\title{
Time-Frequency MUSIC Encephalon Source Localization Algorithm in Impulsive Stable Distribution Noise
}

\author{
WANG Hai-bin 1, a,LI Peng2,b,XIE Huicheng2,c,LONG \\ Jun-bo2,d ,ZHA Dai-feng 2,e \\ 1College of Information Science and Technology Jiujiang University, \\ Jiujiang Jiangxi 332005 China \\ 2College of Electronic and Engineering Jiujiang University, Jiujiang \\ Jiangxi 332005 China \\ awanghaibin00@163.com,b47071565@qq.com , \\ c20205161@qq.com,d18488870@qq.com,e185840922@qq.com,
}

\begin{abstract}
Magnetoencephalograph spatial Time-Frequency Multiple Signal Classification (MEG-TF-MUSIC)can work well in essential and sensor noises environment, but a pulse noise exist in MEG array processing data, they can be described by $\alpha$ stable distribution. The performance of MEG-TF-MUSIC degenerate in $\alpha$ stable distribution environment, Hence, we define the fractional lower order moments spatial time-frequency distribution matrix (FLOM-STFDM) concept based on fractional lower order covariance, and MEG spatial Time-Frequency Fractional Lower order Multiple Signal Classification(FLO-MEG-TF-MUSIC) method in which STFDM is replaced by FLOM-STFDM is proposed, procedures of algorithm is introduced in this paper, it can effectively reduce he source location estimation mean square error (MSE). Simulations show that the technique demonstrate the advantages than MEG-TF-MUSIC in $\alpha$ stable distribution environment, is robust.

Keywords: Magnetoencephalograph; Source localization; Alpha( $\alpha$ ) stable distribution; Multiple Signal Classification

\section{Introduction}

Magnetoencephalograph(MEG) is a ideal tool of human neural activity analysis, brain source (lesions) location method based on MEG has been intensively researched in recent years. Multiple signal classification (MUSIC) algorithm is a kind of noise subspace algorithm and is usually applied to the source and target positioning, in the first place, it is applied to EEG source localization in the literature[1], and EEG-MUSIC source localization algorithm is proposed. Then the improved R-MUSIC, S-MUSIC, IES-MUSIC and RAP MUSIC algorithm based on MUSIC have been proposed, The calculation of these
\end{abstract}


methods in the monophyletic positioning is the same, but RAP MUSIC has the obvious advantage when they are calculated the multi-source location. The actual brain magnetic signal is a non-stationary signal, and time-frequency distribution is a powerful analysis tool for non-stationary signal. Literature [2] introduces the time-frequency distribution matrices in the signal processing and puts forward the concept of spatial time-frequency for non-stationary signals. form spatial time-frequency distribution matrix, The STF-MUSIC estimation method based on time-frequency averageness in which the PWVD time-frequency distribution is used as the spatial time-frequency distribution matrix is proposed in literature [3], effectively improve the estimate resolution, Signal-to-noise ratio(SNR) can be improved $\mathrm{L}$ times than the traditional MUSIC method ( $\mathrm{L}$ is the length of the rectangular window of PWVD). Subsequently, TF-MUSIC is applied to The error calibration of radar matrix signal and MEG field ${ }^{[4-5]}$.

In general, MEG-TF-MUSIC algorithm is regarded as gaussian noise in dealing with the false component (noise) of the EEG data, this assumption in most cases is acceptable, because most researchers average the data for many times before analyzed the experiment, set a threshold value and make it pass a band-pass filter , but it will cause the inevitable loss of data information, hence, the signal processing method based on unequal MEG data is necessary. The noise is usually impulsive in unequal MEG data, for example, the acceleration noise impact, hypoxia and other special test, its strength is far greater than the source potential, and is not belong to the gaussian distribution, but we can described as $\alpha$ stable distribution $^{[6-7]}$

A new method of clutter identification is proposed in view of the $\alpha$ stable distribution clutter in literature [6], It establish the corresponding relationship between $\alpha$ stable distribution parameters and the clutter noise, and can identify several types, the method establish normalization radar echo characteristic function, so we can use $\alpha$ stable distribution theory to estimate radar clutter frequency spectrum. The traditional frequency spectrum estimation algorithm based on second order statistics (SOS) fail in $\alpha$ distributed environmen, therefore, frequency spectrum estimation methods in view of the stable distribution have been proposed, FLOS - direct method, FLOS - indirect method, FLOS - Welch method[7], autoregressive (AR) model spectrum estimation method [8], etc. We substitute the second order correlation with the fractional lower order covariance(FLOC), get the fractional lower order covariance matrix, and apply the matrix decomposition method, put forward three kinds of new method for radar echo frequency spectrum estimation: FLOC - Pisarenko, FLOC Esprit and FLOC - Music method. Three kinds of new method and traditional method based on second order statistics are compared in the paper, the computer simulation shows that the traditional method fails, the proposed method can work under the $\alpha$ stable distribution environment, can also work in gaussian noise environment, has a certain toughness.

\section{MEG-TF-MUSIC algorithm}


The coordinates $X, Y$ and $Z$ are expressed as dipole source location, let us define sensor electrode as $\mathrm{M}$, source signal as $\mathrm{P}$ and $M>P$. The received signal of $M$ sensor at the $t$ moment can be represented as $y(t)=\left[y_{1}(t), y_{2}(t), \cdots, y_{M}(t)\right]^{T}$, P dipole source signals are represented as $s(t)=\left[s_{1}(t), s_{2}(t), \cdots, s_{P}(t)\right]^{T}, x_{1}(t), s_{2}(t), \cdots, s_{P}(t)$ are the source locations, $M \times 2 P$ lead electric field matrix of $\mathrm{P}$ dipole as follows $G=\left[G\left(x_{1}\right), G\left(x_{2}\right), \cdots, G\left(x_{R}\right)\right]$, We also define a $2 P \times P$ orientation matrix of $\mathrm{P}$ dipole that expresses as $M=\operatorname{diag}\left[M_{1}, M_{1}, \cdots, M_{P}\right]$, the received vector at $\mathrm{t}$ moment is defined as

$$
\begin{gathered}
y(t)=[G M(t)] s(t)+n(t)=A(\Theta) s(t)+n(t) \\
\text { Among } A(\Theta) \text { is a } M \times P \quad \text { directional matrix , let } \\
A(\Theta)=\left[a\left(\theta_{1}\right), a\left(\theta_{2}\right), \cdots, a\left(\theta_{P}\right)\right], \quad \theta \quad \text { is hybrid parameter , } \\
n(t)=\left[n_{1}(t), n_{2}(t), \cdots, n_{M}(t)\right]^{T} \text { is the noise vector of } \alpha \text { stable distribution at }
\end{gathered}
$$
$t$ moment.

The Cohen-class time-frequency distribution of the type (1) can be described as:

$$
C_{y}(t, f)=\int_{-\infty}^{+\infty} \int_{-\infty}^{+\infty} \Phi(v-t, \xi-f) \cdot\left[\int_{-\infty}^{+\infty} y(v+\tau / 2) y^{*}(v-\tau / 2) e^{-2 \pi j \xi \tau} d \tau\right] d v d \xi
$$

Here, $\Phi(v-t, \xi-f)$ is the kernel function, when Signal and noise is not relevant we can get $C_{s n}(t, f)=0, C_{n s}(t, f)=0$, type (2) can be simplified as:

$$
\begin{array}{r}
C_{y}(t, f)=(G M) C_{s}(t, f)\left(M^{T} G^{T}\right)+C_{s n}(t, f)+C_{n s}(t, f)+C_{n n}(t, f) \\
=(G M) C_{s}(t, f)\left(M^{T} G^{T}\right)+C_{n n}(t, f)
\end{array}
$$

$C_{s}(t, f)$ is the Cohen-class time-frequency distribution of the source signals, For the source signals of disparate frequency characteristics, for example, two frequency-modulated signal is shown in figure 1, the time- frequency distribution is different, the figure 2 is their PWVD, The core region $\Omega$ of each source signals in the time-frequency distribution are selected, the signal 1 is $\Omega_{1}$ and the signal 1 
is $\Omega_{2}$, after averaging in time-frequency domain, the average time-frequency distribution of the source can be pressed as

$$
\Psi_{y}=\iint_{\Omega} C_{y}(t, f) d t d f=A(\Theta) C_{s}(t, f)\left(A(\Theta)^{T}\right)+\sigma_{n}^{2} I
$$

The noise's characteristic vector are get when $\Psi_{y}$ is carried out the feature decomposition, the type (6) is used as a cost function and EEG inverse problem is applied in the method, the corresponding largest $J(i)$ is the space position of the source when the grid position of the brain model is searched.

$$
J(i)=1 / \lambda_{\min }\left\{a(\theta)^{T} Z_{n} Z_{n}^{T} a(\theta), a(\theta)^{T} a(\theta)\right\}
$$

\section{FLO-MEG-TF-MUSIC algorithm}

When the noise in type (1) is $\alpha$ stable distribution, its time-frequency distribution degenerate, the cost function (6) will not be available, and therefore the traditional EEG -TF-MUSIC must be improved. we defined the fractional lower order Cohen-class time-frequency distribution(FLO-CTFD) of the signal $y(t)$ as

$C_{y}^{F L O}(t, f)=\frac{1}{2 \pi} \int_{-\infty}^{+\infty} \int_{-\infty}^{+\infty} \Phi(v-t, \xi-f)\left[\int_{-\infty}^{+\infty} y^{\langle P\rangle}(v+\tau / 2) y^{-\langle P\rangle}(v-\tau / 2) e^{-2 \pi \xi \xi \tau} d \tau\right] d v d \xi$

$C_{s}^{F L O}(t, f)=\frac{1}{2 \pi} \int_{-\infty}^{+\infty} \int_{-\infty}^{+\infty} \Phi(v-t, \xi-f)\left[\int_{-\infty}^{+\infty}\left\langle{ }^{\langle P\rangle}(v+\tau / 2) s^{-\langle P\rangle}(v-\tau / 2) e^{-2 \pi j \xi \tau} d \tau\right] d v d \xi\right.$

Here, $C_{y}^{F L O}(t, f)$ is the FLO-CTFD of Mixed signal and $C_{s}^{F L O}(t, f)$ is the FLO-CTFD of the source signal. The time-frequency distribution matrices for $C_{y}^{F L O}(t, f)$ can be written as

$C_{y}^{F L O}(t, f)=(G M) C_{s}^{F L O}(t, f)\left(M^{T} G^{T}\right)+C_{s n}^{F L O}(t, f)+C_{n s}^{F L O}(t, f)+C_{n n}^{F L O}(t, f)$

And $C_{s n}^{F L O}(t, f)=\frac{1}{2 \pi} \int_{-\infty}^{+\infty} \infty_{-\infty}^{+\infty} \Phi(v-t, \xi-f)\left[\int_{-\infty}^{+\infty} s^{\langle P>}(v+\tau / 2) n^{-<P>}(v-\tau / 2) e^{-2 \pi j \xi \tau} d \tau\right] d v d \xi$

$C_{n s}^{F L O}(t, f)=\frac{1}{2 \pi} \int_{-\infty}^{+\infty} \int_{-\infty}^{+\infty} \Phi(v-t, \xi-f)\left[\int_{-\infty}^{+\infty} n^{\langle P\rangle}(v+\tau / 2) s^{<P>}(v-\tau / 2) e^{-2 \pi \xi \xi \tau} d \tau\right] d v d \xi$

$C_{n n}^{F L O}(t, f)=\frac{1}{2 \pi} \int_{-\infty}^{+\infty} \int_{-\infty}^{+\infty} \Phi(v-t, \xi-f)\left[\int_{-\infty}^{+\infty} n^{\langle P>}(v+\tau / 2) n^{-<P>}(v-\tau / 2) e^{-2 \pi j \xi \tau} d \tau\right] d v d \xi$

Because the signal $s(t)$ and the stable distribution noise $n(t)$ is uncorrelated, the type(9) can be simplified as 
$C_{y}^{F L O}(t, f)=(G M) C_{s}^{F L O}(t, f)\left(M^{T} G^{T}\right)+C_{n n}^{F L O}(t, f)$

We can average the core region of each source signals in time-frequency distribution, the signal 1 is $\Omega_{1}$ and signal 2 is $\Omega_{2}$ in Figure 2, the averaged FLO-CTFD of the source signal 1 and source signal 2 can be written as $\Psi_{y_{1}}^{F L O}=\iint_{\Omega_{1}} C_{y_{1}}^{F L O}(t, f) d t d f=A(\Theta) \Psi_{s_{1}}^{F L O}\left(A(\Theta)^{T}\right)+\sigma_{n}^{2} I$

$$
\Psi_{y_{2}}^{F L O}=\iint_{\Omega_{2}} C_{y_{2}}^{F L O}(t, f) d t d f=A(\Theta) \Psi_{s_{2}}^{F L O}\left(A(\Theta)^{T}\right)+\sigma_{n}^{2} I
$$

Here, $\Psi_{s_{1}}^{F L O}=\iint_{\Omega} C_{s_{1}}^{F L O}(t, f) d t d f, \quad \Psi_{s_{2}}^{F L O}=\iint_{\Omega} C_{s_{2}}^{F L O}(t, f) d t d f, \quad \Psi_{y_{1}}^{F L O}, \Psi_{s_{1}}^{F L O}, \Psi_{y_{2}}^{F L O}$ and $\Psi_{s_{2}}^{F L O}$ are $P_{\Omega} \times P_{\Omega}$ matrix. We define the function of the brain source localization in the fractional lower order noise environment as:

$$
J(i)=1 / \lambda_{\min }\left\{a(\theta)^{T} Z_{N}^{F L O}\left(Z_{N}^{F L O}\right)^{T} a(\theta), a(\theta)^{T} a(\theta)\right\}
$$

In order to calculate the position of every source signal, we pursue the decomposition of $\Psi_{y_{1}}^{F L O}$ and get noise eigenvectors $Z_{N_{1}}^{F L O}=\left[u_{P_{R_{1}}+1}, \cdots, u_{P_{P_{1}}+M}\right]$, then, apply EEG inverse problem method with a cost function type(16), each location in the grid of the brain model is searched, the corresponding largest point is the spatial position of the source signal 1.the same method to signal 2, $\Psi_{y_{2}}^{F L O}$ is decompose andThe feature vectors is $Z_{N_{2}}^{F L O}=\left[u_{P_{\Omega_{2}}+1}, \cdots, u_{P_{\Omega_{2}}+M}\right]$.

\section{COMPUTER SIMULATION}

The head model is three layers concentric sphere model in this paper and shown in figure 1 , the centre position of sphere brain is $(0,0,0)$ and the radius is 0.88 , the skull radius is 0.92 , the radius of the scalp is 1 , conductivity respectively are 1 , $1 / 80,1$, the electrode is shown in figure 2 , the standard placed 10-20 model and 81 electrodes, the source signal of simulation are two FM signals, such as type (18), type (19), here $a=0.004, \omega=1.72, \quad c=-0.015, \quad n=250$. We assume that the position of the signal source 1 is $(1.0,4.0,-6.5)$, and the position of the signal source 2 is $(1.0,5.0,-8.0)$.

$$
s_{1}=\exp \left[-a(n-130)^{2}+j c(n-130)^{2}+j \omega(n-130)\right]
$$




$$
s_{2}=\exp \left[-a(n-100)^{2}+j c(n-100)^{2}+j \omega(n-100)\right]
$$

8)

The received relative potential data of the source signal in the left side of the channel and normalized spectrum are shown in figure (3) when there are not any noise, figure (4) is the PWVD of the observation data.
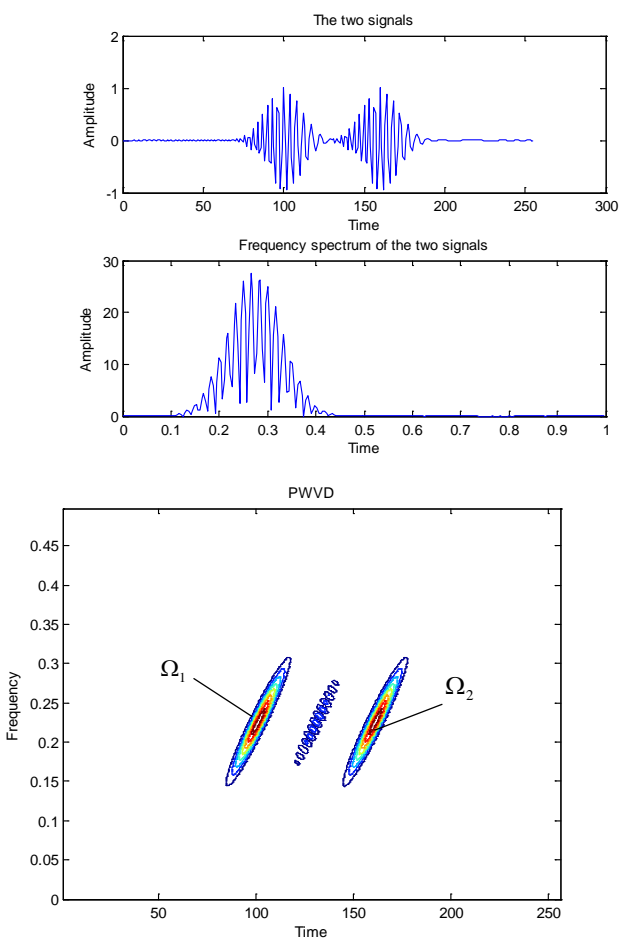

Fig.1 Observed signals and its frequency spectrum

Fig.2 the PWVD of the observed signals

When the noise is the stable distribution of noise, we use the generalized SNR (GNSR) instead of the traditional signal-to-noise ratio, the GNSR is defined as GNSR $=10 \log \left(E\left\{\left.y(t)\right|^{2}\right\} / \gamma^{\alpha}\right)$, the mixed GNSR $=20 \mathrm{~dB} S \alpha S$ stable distribution white noise and signal is shown in figure 3.In order to compare the performance of the MEG-RAP-MUSIC algorithm and the proposed MEG-FLO-TF-MUSIC algorithm, we define the hybrid mean square error (MSE) of the monophyletic positioning as:

$$
M S E=\frac{1}{2 K} \sum_{k=1}^{K}\left(\hat{X}_{1}-X_{1}\right)^{2}+\frac{1}{2 K} \sum_{k=1}^{K}\left(\hat{Y}_{1}-Y_{1}\right)^{2}+\frac{1}{2 K} \sum_{k=1}^{K}\left(\hat{Z}_{1}-Z_{1}\right)^{2}
$$

Here, $\mathrm{K}$ is Monte Carlo experiment times. 

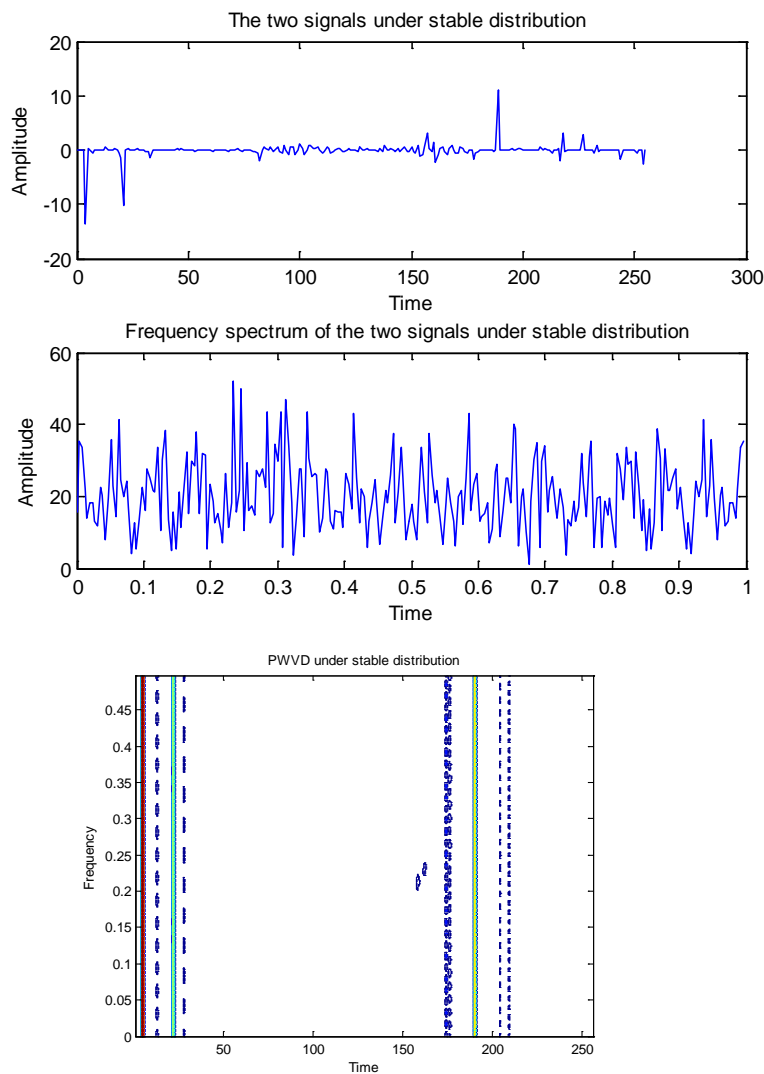

Fig.3 Observed signals and its frequency

Fig.4 The PWVD of the observed signals in spectrum in SaS stable distribution noise $S \alpha S$ stable distribution noise

We carried out PWVD computing on the observation signal containing $S \alpha S$ noise, as shown in figure 4, the conventional PWVD method complete failure, and the improved FLO-PWVD has good performance, the result of FLO-PWVD is shown in figure 5 , In order to further compare two algorithms, we change the GNSR of the $S \alpha S$ stable distribution noise from $14 \mathrm{~dB}$ to $24 \mathrm{~dB}$, the MES of MEG-FLO-TF-MUSIC[9] and the proposed FLOM-EEG-RAP-MUSIC algorithms are shown in figure 6, it show that the FLO-MEG-TF-MUSIC algorithm on MSE is better than that of FLOM-EEG-RAP-MUSIC algorithms. 

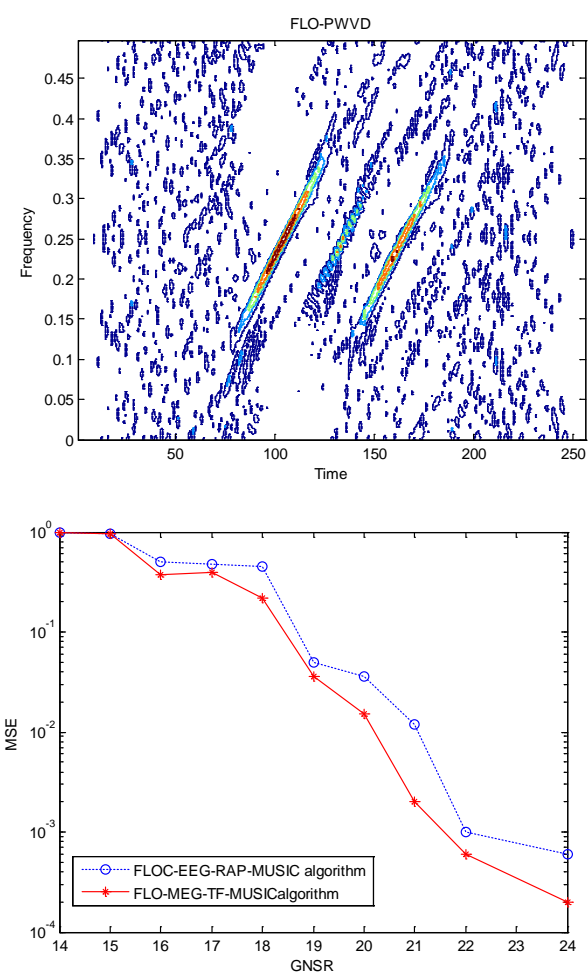

Fig.5 The FLO-PWVD of the observed signals under

Fig.6 Comparing Source localization under $S \alpha S$ stable distribution noise different mean square error (MSE)

\section{DISCUSSION}

The peaks in the electrical impulse noise can be described by $\alpha$ stable distribution, traditional Cohen- class time-frequency distribution fail in calculation under the noisy environment, therefore, we put forward a new FLO-EEG-TF -MUSIC algorithm. fractional lower order Cohen time-frequency distribution is used instead of the traditional Cohen-class time-frequency distribution in our method, then we calculate spatial time-frequency where are the core region of the source signal time-frequency distribution, and get fractional lower order spatial time-frequency matrix, then, the fractional lower order spatial time-frequency matrix is used to calculate the source locations.The proposed new algorithm not only can improve the positioning resolution, can work under strong impulse noise, also is suitable for gaussian noise, it has a certain toughness and the method has a certain practical significance in special cases of the brain source location. 


\section{Acknowledgements}

This work was financially supported by Natural Science Foundation of China (61261046), Jiangxi province natural science foundation of China(20142BAB207006), Science and technology project of provincial education department of jiangxi (GJJ11621, GJJ11245, GJJ11244, GJJ14739, GJJ14721), Science and technology project of jiujiang university (2013KJ01, 2013KJ02).

\section{References}

[1] J. C. Mosher, P. S. Lewis, and R. M. Leahy, Multiple Dipole Modeling and Localization from Spatio-Temporal MEG Data[J],IEEE Transaction Biomedical Engineering, 1992. 39:541-557.

[2] Belouchrani.A, M.Amin. Blind source separation based on time-frequency signal representation[J].IEEE Transaction signal processing,1998,46(11):2888-2897.

[3] Zhang Yimin, Mu weifeng,Amin.M.G.Subspace Analysis of Spatial Time-Frequency Distribution Matrices[J].IEEE Transactions on signal processing,2001,49(4):747-759.

[4] Mohmed.K,adel.B,Karim A.M.Performance analysis for time-frequency MUSIC algorithm in presence of both additive noise and array calibration errors[J] , EURASIP Journal on Advances in Signal Processing,2012,4(94):1-11

[5] K.Sekihara,S.Nagarajan,D.Poeppel,etc. Time-Frequency MEG-MUSIC Algorithm[J], IEEE Transactions On Medical Imaging, 1999. 18(1):92-97.

[6] M.shao, C.L.Nikias. Singnal Processing with Fractional Lower Order Moments Instable Processed and Their Applications[J].proceedings of the IEEE. 1993， 81(7):986-1010.

[7]LING Zheng-jian, ZHA Dai-feng, SHENG Jian. Blind Estimation of Evoked Potentials Based on Covariations in Non-Gaussian Noise[J],Journal of Biomedical Engineering, 2010, 04: 727-730.

[8] WANG Hai-bin, ZHA Dai-feng, LONG Jun-bo.Spatial Time-frequency DOA Estimation Under $\alpha$ Stable Distribution Noise[J], Computer Engineering, 2012,38(2):284-287. 